\title{
Impact of body mass index on real-world outcomes of rivaroxaban treatment in Japanese patients with non-valvular atrial fibrillation
}

\author{
Yuji Murakawa' ${ }^{1}$ Takanori Ikeda $^{2} \cdot$ Satoshi Ogawa ${ }^{3} \cdot$ Takanari Kitazono $^{4} \cdot$ Jyoji Nakagawara $^{5,6} \cdot$ Kazuo Minematsu $^{6,7}$. \\ Susumu Miyamoto ${ }^{8} \cdot$ Yasuhiro Hayashi $^{9} \cdot$ Yoko Kidani $^{9} \cdot$ Yutaka Okayama $^{10} \cdot$ Toshiyuki Sunaya $^{11}$. Shoichiro Sato ${ }^{10}$. \\ Satoshi Yamanaka ${ }^{9}$
}

Received: 18 November 2019 / Accepted: 13 March 2020 / Published online: 6 April 2020

(c) The Author(s) 2020

\begin{abstract}
This sub-analysis of the XAPASS, a prospective, single-arm, observational study, aimed to evaluate relationships between body mass index (BMI) and safety (major bleeding and all-cause mortality) and effectiveness [stroke/non-central nervous system (non-CNS) systemic embolism (SE)/myocardial infarction (MI)] outcomes in Japanese patients with non-valvular atrial fibrillation (NVAF) receiving rivaroxaban. Patients were categorized according to BMI $\left(\mathrm{kg} / \mathrm{m}^{2}\right)$ as underweight $(<18.5)$, normal weight $(18.5$ to $<25)$, overweight $(25$ to $<30)$, or obese $(\geq 30)$. In total, 9578 patients with NVAF completed the 1-year follow-up and were evaluated; of these, 7618 patients had baseline BMI data. Overall, 542 (5.7\%), 4410 (46.0\%), 2167 (22.6\%), and 499 (5.2\%) patients were underweight, normal weight, overweight, and obese, respectively. Multivariable Cox regression analysis demonstrated that none of the BMI categories were independent predictors of major bleeding whereas being underweight was independently associated with increased all-cause mortality [hazard ratio (HR) $3.56,95 \%$ confidence interval (CI) 2.40-5.26, $p<0.001]$. The incidence of stroke/non-CNS SE/MI was higher in patients who were underweight than in those of normal weight (HR 2.11, 95\% CI 1.20-3.70, $p=0.009$ ). However, in multivariable analyses, being underweight was not identified as an independent predictor of stroke/non-CNS SE/MI (HR 1.64, 95\% CI 0.90-2.99, $p=0.104$ ). In conclusion, the high incidence of thromboembolic events and all-cause mortality in patients who were underweight highlights that thorough evaluation of disease status and comorbidities may be required in this population.
\end{abstract}

Keywords Atrial fibrillation $\cdot$ Body mass index $\cdot$ Stroke $\cdot$ Rivaroxaban

Electronic supplementary material The online version of this article (https://doi.org/10.1007/s00380-020-01587-z) contains supplementary material, which is available to authorized users.

Yuji Murakawa

murakawa@med.teikyo-u.ac.jp

1 The 4th Department of Internal Medicine, Teikyo University School of Medicine, Mizonokuchi Hospital, 5-1-1, Futago, Takatsu-ku, Kawasaki, Kanagawa 213-8507, Japan

2 Department of Cardiovascular Medicine, Toho University Graduate School of Medicine, Tokyo, Japan

3 International University of Health and Welfare Mita Hospital, Tokyo, Japan

4 Department of Medicine and Clinical Science, Graduate School of Medical Sciences, Kyushu University, Fukuoka, Japan

5 Osaka Namba Clinic, Osaka, Japan
6 National Cerebral and Cardiovascular Center, Suita, Osaka, Japan

7 Iseikai Medical Corporation, Osaka, Japan

8 Department of Neurosurgery, Kyoto University Graduate School of Medicine, Kyoto, Japan

9 Medical Affairs Thrombosis, Medical Affairs, Bayer Yakuhin, Ltd., Osaka, Japan

10 Pharmacovigilance Monitoring and Medical Governance, Medical Affairs, Bayer Yakuhin, Ltd., Osaka, Japan

11 Research and Development Japan/Data Sciences and Analytics/Statistics and Data Insights, Bayer Yakuhin, Ltd., Osaka, Japan 


\section{Introduction}

Direct oral anticoagulants (DOACs) such as rivaroxaban offer an alternative to vitamin $\mathrm{K}$ antagonists as anticoagulant treatments for stroke prevention in non-valvular atrial fibrillation (NVAF) [1]. Over the past decade, there has been a growing interest in the relationship between body mass index (BMI) and clinical outcomes in patients with atrial fibrillation (AF). Although obesity is a risk factor for $\mathrm{AF}$, some studies have demonstrated that among patients with $\mathrm{AF}$, mortality is lower in those with obesity than in those of normal weight, a phenomenon known as the obesity paradox [2-4]. However, the impact of being underweight on the incidence of thromboembolism and bleeding outcomes has been difficult to assess because patients who are underweight are often underrepresented in global or Western clinical trials and observational studies. Moreover, many trials do not consider differences in body composition; for example, Western patients tend to be taller and have higher body weight than Asian patients. A recent meta-analysis, which included Asian patients, demonstrated that being underweight was associated with an increased risk of stroke or systemic embolism (SE) [5]; however, other studies showed no associations between being underweight and the incidence of thromboembolism $[6,7]$. These inconsistent data suggest a need to further examine the impact of BMI on outcomes in patients being treated for stroke prevention in AF. In the present subanalysis of the Xarelto Post-Authorization Safety \& Effectiveness Study in Japanese Patients with Atrial Fibrillation (XAPASS), we examined the relationship between BMI and clinical outcomes in Japanese patients with NVAF using the XAPASS 1-year follow-up data.

\section{Materials and methods}

\section{Study design}

The XAPASS (Clinicaltrials.gov: NCT01582737) is a realworld, prospective, open-label, single-arm, observational, post-authorization cohort study conducted in Japan. The study design has been described previously [8]. Briefly, the standard observation period for each patient is 2 years with data collection at 6 months, 1 year, and 2 years after the initiation of rivaroxaban treatment. After the completion of the standard observation period, follow-up investigations will be conducted for a maximum of 5 years. The study was approved by the Ministry of Health, Labour, and Welfare in Japan and was carried out in accordance with the standards for Good Post-marketing Study Practice provided by this ministry. Individual consent and institutional approval of ethical standards in accordance with the Declaration of Helsinki are not necessary in activities and research for the safety surveillance, such as signal detection and prospective cohort studies.

\section{Patients}

In total, 11,308 Japanese patients with NVAF were enrolled in the XAPASS between April 2012 and June 2014. The current sub-analysis included 9578 patients who had completed at least 11 months of the 1-year follow-up, discontinued rivaroxaban treatment within 1 year, or were lost to follow-up within 1 year [9]. The patients were divided into four BMI $\left(\mathrm{kg} / \mathrm{m}^{2}\right)$ categories according to the World Health Organization technical report series: underweight $(<18.5)$, normal weight $(18.5$ to $<25)$, overweight $(25$ to $<30)$, and obese $(\geq 30)[10]$.

\section{Treatment}

Patients received oral rivaroxaban at a dosage of either $15 \mathrm{mg}$ once daily (o.d.) or $10 \mathrm{mg}$ o.d., at the discretion of the treating physician. These dosages are approved in Japan for patients with creatinine clearance $(\mathrm{CrCl}) \geq 50$ and $<50 \mathrm{ml} /$ min, respectively.

\section{Study outcomes}

The primary safety outcome was any bleeding. Major bleeding and intracranial hemorrhage were recorded as the components. Major bleeding was defined according to the International Society on Thrombosis and Haemostasis criteria [11]. The primary effectiveness outcome was a composite of stroke (hemorrhagic or ischemic), non-central nervous system (non-CNS) SE, and myocardial infarction (MI). All the outcomes were defined previously $[8,9]$. Stroke and ischemic stroke were recorded as individual outcomes. Transient ischemic attack (TIA) was not included in the stroke endpoint.

\section{Statistical analysis}

Patient characteristics at baseline were summarized by frequencies and percentages for categorical data. Survival curves were estimated by the Kaplan-Meier method. Univariable and multivariable Cox regression analyses were performed to estimate hazard ratios (HRs) of outcomes within the four BMI categories. The following variables at the study enrollment were included as explanatory variables: age, sex, $\mathrm{CrCl}$, initial dose, hypertension, diabetes mellitus, congestive heart failure, prior ischemic stroke/TIA, vascular disease, hepatic dysfunction, and oral antiplatelet 
use considering medical interest, multicollinearity, and data availability. All statistical analyses were performed using SAS version 9.2 or higher (SAS Institute Inc., Cary, NC).

\section{Results}

\section{Patients}

In total, 9578 patients with NVAF completed the 1-year follow-up and were evaluated and 7618 patients had baseline BMI data. Overall, 542 (5.7\%), 4,410 (46.0\%), 2,167 (22.6\%), and 499 (5.2\%) patients were underweight, normal weight, overweight, and obese, respectively; demographic characteristics varied across the BMI categories (Table 1). Compared with patients of normal weight, a numerically higher proportion of those categorized as underweight were female or $\geq 75$ years of age. Among those who were underweight, normal weight, overweight, and obese, $62.9 \%$, $29.1 \%, 11.6 \%$, and $6.2 \%$ had $\mathrm{CrCl}<50 \mathrm{ml} / \mathrm{min}$, respectively. The proportion of patients with comorbid hypertension and diabetes was highest among patients with obesity and lowest among those who were underweight. $\mathrm{CHADS}_{2}$, $\mathrm{CHA}_{2} \mathrm{DS}_{2}$-VASc, and modified HAS-BLED scores were similar across BMI groups (Table 1).

\section{Treatment period and rivaroxaban dosage}

The mean ( \pm standard deviation) treatment duration was 259 $( \pm 140), 301( \pm 117), 307( \pm 114)$, and $306( \pm 115)$ days in patients who were underweight, normal weight, overweight, and obese, respectively. Among those with $\mathrm{CrCl}<50 \mathrm{ml} /$ $\min , 8.1 \%$ (44/542), 3.0\% (134/4410), 1.0\% (22/2167), and $0.6 \%$ (3/499) in the underweight, normal weight, overweight, and obese group, respectively, received rivaroxaban $15 \mathrm{mg}$ o.d. (overdose) (Supplementary Fig. 1). Among those with $\mathrm{CrCl} \geq 50 \mathrm{ml} / \mathrm{min}, 17.9 \%$ (97/542), 23.9\% (1054/4410), $31.0 \%$ (672/2167), and 32.5\% (162/499) in the underweight, normal weight, overweight, and obese group, respectively, received rivaroxaban $10 \mathrm{mg}$ o.d. (underdose) (Supplementary Fig. 1).

\section{Safety and effectiveness outcomes}

Safety and effectiveness outcome event rates are shown in Table 2 and Supplementary Table 1. Major bleeding event rates per 100 patient-years were 1.87 in the normal weight (reference) group, 2.19 in the underweight group, 1.71 in the overweight group, and 1.63 in the obese group (Table 2). The cumulative incidences of major bleeding were similar across all groups when normal BMI was used as the reference [HR 1.15, 95\% confidence interval (CI) $0.57-2.30, p=0.692$ for patients who were underweight;
HR $0.92,95 \%$ CI $0.61-1.40, p=0.700$ for patients who were overweight; HR $0.88,95 \%$ CI $0.40-1.91, p=0.740$ for patients who were obese] (Fig. 1a and Table 3). Multivariable analysis demonstrated no independent associations between BMI categories and major bleeding (Table 3).

All-cause mortality per 100 patient-years was 1.91 in the normal weight (reference) group, 10.66 in the underweight group, 1.76 in the overweight group, and 0.93 in the obese group (Table 2). The univariable analysis demonstrated that, compared with patients of normal weight, all-cause mortality was significantly higher in patients who were underweight (HR 5.51, 95\% CI 3.78-8.01, $p<0.001$ ) and similar in those who were overweight (HR $0.92,95 \%$ CI $0.61-1.39, p=0.705$ ) or obese (HR 0.49 , 95\% CI 0.18-1.33, $p=0.161$ ) (Table 3). Multivariable analyses with normal weight as the reference demonstrated an independent association between being underweight and all-cause mortality (HR 3.56, 95\% CI 2.40-5.26, $p<0.001$ ) (Table 3). Being overweight (HR 1.19, 95\% CI $0.77-1.83, p=0.427$ ) or obese (HR $0.74,95 \%$ CI $0.27-2.05, p=0.562$ ) was not associated with increased all-cause mortality (Table 3 ).

The incidence per 100 patient-years of stroke/nonCNS SE/MI was 1.69 in the normal weight (reference) group, 3.67 in the underweight group, 1.77 in the overweight group, and 1.63 in the obese group (Table 2). Compared with patients of normal weight, the cumulative incidence of stroke/non-CNS SE/MI was significantly higher in patients who were underweight (HR 2.11, 95\% CI $1.20-3.70, p=0.009)$ and similar in those who were overweight (HR 1.06, 95\% CI $0.69-1.61, p=0.800$ ) or obese (HR 0.97, 95\% CI 0.44-2.12, $p=0.940$ ) (Fig. 1b and Table 3). Multivariable analysis with normal BMI as the reference identified no independent associations between any of the BMI categories and the incidence of stroke/ non-CNS SE/MI (Table 3).

\section{Adverse events leading to all-cause mortality}

Overall, $8.1 \%, 1.6 \%, 1.5 \%$, and $0.8 \%$ of patients in the underweight, normal weight, overweight, and obese group, respectively, experienced an adverse event that led to death (all-cause mortality) (Supplementary Table 1). In particular, $0.37 \%, 0.18 \%, 0.37 \%$, and $0 \%$ of patients in the underweight, normal weight, overweight, and obese group, respectively, died as a result of adverse drug reactions (Supplementary Table 1). Compared with those in the normal, overweight, and obese categories, there was a trend towards a greater proportion of patients who were underweight dying from cardiac disorders, cancer, and respiratory disorders (Table 4). 
Table 1 Baseline characteristics

\begin{tabular}{|c|c|c|c|c|}
\hline & \multicolumn{4}{|l|}{ BMI $\left(\mathrm{kg} / \mathrm{m}^{2}\right)$} \\
\hline & $\begin{array}{l}\text { Underweight }(<18.5) \\
(n=542)\end{array}$ & $\begin{array}{l}\text { Normal }(18.5 \text { to }<25) \\
(n=4410)\end{array}$ & $\begin{array}{l}\text { Overweight }(25 \text { to < } 30) \\
(n=2167)\end{array}$ & Obese $(\geq 30)(n=499)$ \\
\hline BMI, mean $( \pm \mathrm{SD}), \mathrm{kg} / \mathrm{m}^{2}$ & $17.1( \pm 1.1)$ & $22.2( \pm 1.7)$ & $26.9( \pm 1.4)$ & $33.4( \pm 3.8)$ \\
\hline \multicolumn{5}{|l|}{ Age, years } \\
\hline Mean $( \pm$ SD) & $78.1( \pm 9.0)$ & $73.6( \pm 9.6)$ & $71.3( \pm 9.6)$ & $69.5( \pm 11.0)$ \\
\hline$<75$ & $166(30.6)$ & $2209(50.1)$ & $1274(58.8)$ & $305(61.1)$ \\
\hline$\geq 75$ & $376(69.4)$ & $2201(49.9)$ & $893(41.2)$ & 194 (38.9) \\
\hline Female sex & $287(53.0)$ & $1638(37.1)$ & $704(32.5)$ & $221(44.3)$ \\
\hline \multicolumn{5}{|l|}{$\mathrm{CrCl}, \mathrm{ml} / \mathrm{min}$} \\
\hline Mean $( \pm \mathrm{SD})$ & $47.0( \pm 18.8)$ & $63.0( \pm 28.5)$ & $77.5( \pm 25.6)$ & $97.9( \pm 36.4)$ \\
\hline$<50$ & $341(62.9)$ & $1284(29.1)$ & $252(11.6)$ & $31(6.2)$ \\
\hline$\geq 50$ & $195(36.0)$ & $3091(70.1)$ & $1893(87.4)$ & $461(92.4)$ \\
\hline Unknown & $6(1.1)$ & $35(0.8)$ & $22(1.0)$ & $7(1.4)$ \\
\hline \multicolumn{5}{|l|}{ Comorbidities } \\
\hline Hypertension & $323(59.6)$ & $3195(72.4)$ & $1,804(83.2)$ & 437 (87.6) \\
\hline Diabetes mellitus & $81(14.9)$ & $869(19.7)$ & $596(27.5)$ & $197(39.5)$ \\
\hline Prior ischemic stroke/TIA & $153(28.2)$ & $1032(23.4)$ & $421(19.4)$ & $100(20.0)$ \\
\hline Congestive heart failure & $162(29.9)$ & $1099(24.9)$ & $529(24.4)$ & $131(26.3)$ \\
\hline Hepatic dysfunction & $31(5.7)$ & $254(5.8)$ & $148(6.8)$ & $50(10.0)$ \\
\hline \multicolumn{5}{|l|}{ Type of AF } \\
\hline Paroxysmal & $197(36.3)$ & $1546(35.1)$ & $723(33.4)$ & $143(28.7)$ \\
\hline Persistent & $200(36.9)$ & $1582(35.9)$ & $803(37.1)$ & $192(38.5)$ \\
\hline Permanent & $117(21.6)$ & $1041(23.6)$ & $552(25.5)$ & $141(28.3)$ \\
\hline Other & $0(0)$ & $9(0.2)$ & $9(0.4)$ & $0(0)$ \\
\hline Unknown & $28(5.2)$ & $232(5.3)$ & $80(3.7)$ & $23(4.6)$ \\
\hline \multicolumn{5}{|l|}{$\mathrm{CHADS}_{2}$ score } \\
\hline Mean $( \pm$ SD $)$ & $2.3( \pm 1.4)$ & $2.2( \pm 1.3)$ & $2.2( \pm 1.3)$ & $2.3( \pm 1.2)$ \\
\hline 0 & $58(10.7)$ & $450(10.2)$ & $137(6.3)$ & $16(3.2)$ \\
\hline 1 & $97(17.9)$ & $1,031(23.4)$ & $606(28.0)$ & $121(24.3)$ \\
\hline 2 & $158(29.2)$ & $1,330(30.2)$ & $682(31.5)$ & $156(31.3)$ \\
\hline 3 & $113(20.8)$ & $848(19.2)$ & $389(18.0)$ & $121(24.2)$ \\
\hline 4 & 77 (14.2) & $516(11.7)$ & $236(10.9)$ & $58(11.6)$ \\
\hline 5 & $34(6.3)$ & $197(4.5)$ & $89(4.1)$ & $23(4.6)$ \\
\hline 6 & $5(0.9)$ & $38(0.9)$ & $28(1.3)$ & $4(0.8)$ \\
\hline \multicolumn{5}{|l|}{$\mathrm{CHA}_{2} \mathrm{DS}_{2}$-VASc score } \\
\hline Mean $( \pm S D)$ & $3.8( \pm 1.6)$ & $3.4( \pm 1.6)$ & $3.3( \pm 1.6)$ & $3.5( \pm 1.6)$ \\
\hline 0 & $7(1.3)$ & $123(2.8)$ & $56(2.6)$ & $7(1.4)$ \\
\hline 1 & $39(7.2)$ & $448(10.2)$ & $220(10.2)$ & $36(7.2)$ \\
\hline 2 & $62(11.4)$ & $698(15.8)$ & $439(20.3)$ & $92(18.4)$ \\
\hline 3 & $123(22.7)$ & $1,050(23.8)$ & $515(23.8)$ & $115(23.0)$ \\
\hline 4 & $130(24.0)$ & $1,013(23.0)$ & $454(21.0)$ & $114(22.8)$ \\
\hline 5 & $102(18.8)$ & $629(14.3)$ & $270(12.5)$ & $81(16.2)$ \\
\hline 6 & $51(9.4)$ & $319(7.2)$ & $148(6.8)$ & $37(7.4)$ \\
\hline 7 & $28(5.2)$ & $109(2.5)$ & $49(2.3)$ & $14(2.8)$ \\
\hline 8 & $0(0)$ & $20(0.5)$ & $16(0.7)$ & $3(0.6)$ \\
\hline 9 & $0(0)$ & $1(<0.1)$ & $0(0)$ & $0(0)$ \\
\hline \multicolumn{5}{|l|}{ Modified HAS-BLED score ${ }^{a}$} \\
\hline Mean $( \pm S D)$ & $1.7( \pm 0.9)$ & $1.5( \pm 1.0)$ & $1.4( \pm 1.0)$ & $1.5( \pm 1.0)$ \\
\hline 0 & $31(5.7)$ & $528(12.0)$ & $332(15.3)$ & $77(15.4)$ \\
\hline
\end{tabular}


Table 1 (continued)

\begin{tabular}{lcccc}
\hline \multicolumn{1}{l}{ BMI $\left(\mathrm{kg} / \mathrm{m}^{2}\right)$} & & \\
\cline { 2 - 5 } & $\begin{array}{l}\text { Underweight }(<18.5) \\
(n=542)\end{array}$ & $\begin{array}{l}\text { Normal }(18.5 \text { to }<25) \\
(n=4410)\end{array}$ & $\begin{array}{l}\text { Overweight }(25 \text { to }<30) \\
(n=2167)\end{array}$ & Obese $(\geq 30)(n=499)$ \\
\hline 1 & $215(39.7)$ & $1,776(40.3)$ & $905(41.8)$ & $204(40.9)$ \\
2 & $191(35.2)$ & $1,431(32.4)$ & $650(30.0)$ & $145(29.1)$ \\
3 & $89(16.4)$ & $543(12.3)$ & $226(10.4)$ & $56(11.2)$ \\
4 & $12(2.2)$ & $120(2.7)$ & $46(2.1)$ & $16(3.2)$ \\
5 & $4(0.7)$ & $12(0.3)$ & $1(0.3)$ & $0.2)$ \\
6 & $0(0)$ & $0(0)$ & $0(0)$ & $0(0)$ \\
7 & $0(0)$ & $0(0)$ & $0(0)$ & $0(0)$ \\
8 & $0(0)$ & $768(17.4)$ & $271(12.5)$ & $66(13.2)$ \\
Visit classification & $172(31.7)$ & $3,642(82.6)$ & $1,896(87.5)$ & $433(86.8)$ \\
In-hospital & $370(68.3)$ & & \\
\hline
\end{tabular}

Data are presented as $n(\%)$ unless otherwise indicated

$A F$ atrial fibrillation, $B M I$ body mass index, $\mathrm{CHADS}_{2}$ Congestive heart failure, Hypertension, Age $\geq 75$ years, Diabetes, Prior stroke or TIA, $\mathrm{CHA}_{2} \mathrm{DS}_{2}-\mathrm{VASc}$ Congestive heart failure, Hypertension, Age $\geq 75$ years, Age 65-74 years, Diabetes mellitus, Prior stroke or TIA or Thromboembolism, Sex, Vascular disease (e.g. peripheral artery disease, myocardial infarction or aortic plaque), $\mathrm{CrCl}$ creatinine clearance, HAS- $\mathrm{BLED}$ Hypertension, Abnormal renal and liver function, Stroke, Bleeding, Labile INR, Elderly, Drugs or alcohol, SD standard deviation, TIA transient ischemic attack

aMaximum score is 8 because of the exclusion of the factor "labile international normalized ratio" from the HAS-BLED score

Table 2 Incidences of safety and effectiveness outcomes

\begin{tabular}{|c|c|c|c|c|}
\hline \multirow[b]{4}{*}{ Safety outcome } & \multicolumn{4}{|l|}{ BMI $\left(\mathrm{kg} / \mathrm{m}^{2}\right)$} \\
\hline & Underweight $(<18.5)$ & Normal $(18.5$ to $<25)$ & Overweight $(25$ to $<30)$ & Obese $(\geq 30)$ \\
\hline & \multicolumn{4}{|c|}{ Incidence, events per 100 patient-years (95\% CI) } \\
\hline & $(n=542)$ & $(n=4410)$ & $(n=2167)$ & $(n=499)$ \\
\hline Any bleeding & $11.72(8.33-15.11)$ & $8.42(7.47-9.36)$ & $7.04(5.82-8.26)$ & $5.47(3.23-7.70)$ \\
\hline Major bleeding & $2.19(0.76-3.62)$ & $1.87(1.43-2.30)$ & $1.71(1.12-2.31)$ & $1.63(0.42-2.84)$ \\
\hline Fatal & $0.24(0.00-0.72)$ & $0.11(0.00-0.21)$ & $0.32(0.06-0.58)$ & $0(0)$ \\
\hline Critical organ bleeding & $0.73(0.00-1.55)$ & $0.85(0.56-1.14)$ & $0.96(0.52-1.41)$ & $0(0)$ \\
\hline Intracranial hemorrhage & $0.73(0.00-1.55)$ & $0.77(0.49-1.05)$ & $0.80(0.40-1.21)$ & $0(0)$ \\
\hline Hemoglobin decrease $\geq 2 \mathrm{~g} / \mathrm{dl}$ & $0.73(0.00-1.55)$ & $0.69(0.43-0.96)$ & $0.53(0.20-0.86)$ & $1.16(0.14-2.18)$ \\
\hline $\begin{array}{l}\text { Transfusion of } \geq 2 \text { units of packed } \\
\text { RBC or whole blood }\end{array}$ & $0.48(0.00-1.16)$ & $0.21(0.07-0.36)$ & $0.16(0.00-0.34)$ & $0.23(0.00-0.69)$ \\
\hline All-cause mortality & $10.66(7.51-13.81)$ & $1.91(1.47-2.35)$ & $1.76(1.16-2.36)$ & $0.93(0.02-1.84)$ \\
\hline Effectiveness outcome & $(n=540)$ & $(n=4392)$ & $(n=2161)$ & $(n=497)$ \\
\hline Stroke/non-CNS SE/MI & $3.67(1.81-5.53)$ & $1.69(1.27-2.10)$ & $1.77(1.17-2.38)$ & $1.63(0.42-2.84)$ \\
\hline Stroke & $2.94(1.28-4.60)$ & $1.55(1.15-1.95)$ & $1.61(1.04-2.19)$ & $1.16(0.14-2.18)$ \\
\hline Ischemic stroke & $2.20(0.76-3.64)$ & $1.07(0.74-1.40)$ & $1.07(0.60-1.54)$ & $1.16(0.14-2.18)$ \\
\hline
\end{tabular}

$B M I$ body mass index, $C I$ confidence interval, $C N S$ central nervous system, $M I$ myocardial infarction, $R B C$ red blood cell, $S E$ systemic embolism

\section{Discussion}

The key findings of this study in Japanese patients with NVAF receiving rivaroxaban can be summarized as follows: first, baseline characteristics varied across BMI categories; second, the incidences of stroke/non-CNS SE/MI and all-cause mortality were significantly higher in those who were underweight than in those of normal weight; third, none of the BMI categories emerged as an independent predictor of major bleeding or stroke/ 
a

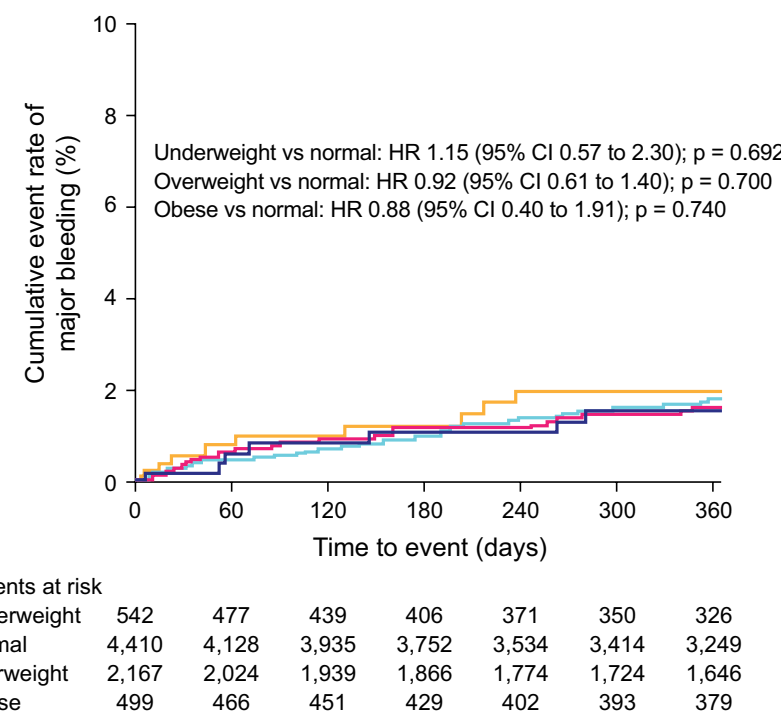

Fig. 1 Kaplan-Meier curves for the cumulative event rate of a major bleeding and $\mathbf{b}$ stroke/non-CNS SE/MI among four BMI categories*. $B M I$ body mass index, $C I$ confidence interval, $C N S$ central nervous b

BMI categories

- Underweight

- Normal

- Overweight

- Obese

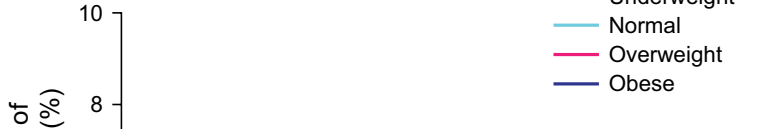

Underweight vs normal: HR 2.11 (95\% Cl 1.20 to 3.70 ); $p=0.009$

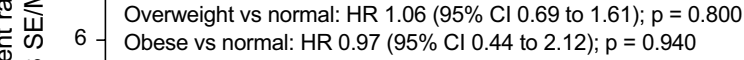

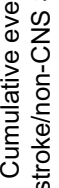

2
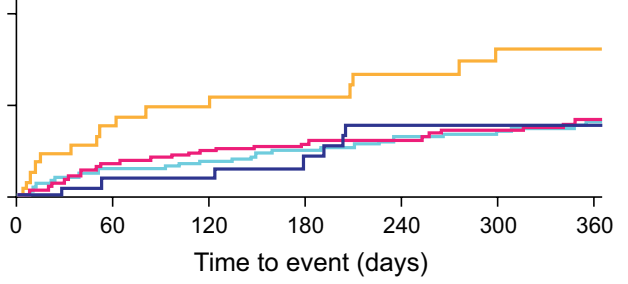

Patients at risk

$\begin{array}{llllllll}\text { Underweight } & 540 & 473 & 437 & 405 & 371 & 349 & 325\end{array}$

$\begin{array}{llllllll}\text { Normal } & 4,392 & 4,106 & 3,918 & 3,735 & 3,522 & 3,405 & 3,241\end{array}$

$\begin{array}{llllllll}\text { Overweight } & 2,161 & 2,016 & 1,930 & 1,859 & 1,767 & 1,720 & 1,641\end{array}$

$\begin{array}{llllllll}\text { Obese } & 497 & 466 & 452 & 430 & 402 & 394 & 380\end{array}$

system, HR hazard ratio, $M I$ myocardial infarction, $S E$ systemic embolism. *BMI categories $\left(\mathrm{kg} / \mathrm{m}^{2}\right)$ : underweight, $<18.5$; normal, 18.5 to $<25$; overweight, 25 to $<30$; and obese, $\geq 30$

Table 3 Risk of major bleeding, all-cause mortality, and stroke/non-CNS SE/MI

\begin{tabular}{|c|c|c|c|c|c|c|c|c|}
\hline \multirow[t]{2}{*}{ Variable $^{\mathrm{a}}$} & \multirow[t]{2}{*}{ Number of events } & \multirow[t]{2}{*}{ Proportion (\%) } & \multicolumn{3}{|c|}{ Univariable analysis } & \multicolumn{3}{|c|}{ Multivariable analysis $^{\mathrm{b}}$} \\
\hline & & & HR & $95 \% \mathrm{CI}$ & $p$ value & HR & $95 \% \mathrm{CI}$ & $p$ value \\
\hline \multicolumn{9}{|l|}{ Major bleeding } \\
\hline Underweight & $9 / 542$ & 1.66 & 1.15 & $(0.57-2.30)$ & 0.692 & 0.97 & $(0.48-1.98)$ & 0.939 \\
\hline Normal & $70 / 4,410$ & 1.59 & 1.00 & Reference & & 1.00 & Reference & \\
\hline Overweight & $32 / 2,167$ & 1.48 & 0.92 & $(0.61-1.40)$ & 0.700 & 0.97 & $(0.63-1.50)$ & 0.906 \\
\hline Obese & $7 / 499$ & 1.40 & 0.88 & $(0.40-1.91)$ & 0.740 & 0.89 & $(0.40-1.97)$ & 0.772 \\
\hline \multicolumn{9}{|c|}{ All-cause mortality } \\
\hline Underweight & $44 / 542$ & 8.12 & 5.51 & $(3.78-8.01)$ & $<0.001$ & 3.56 & $(2.40-5.26)$ & $<0.001$ \\
\hline Normal & $72 / 4,410$ & 1.63 & 1.00 & Reference & & 1.00 & Reference & \\
\hline Overweight & $33 / 2,167$ & 1.52 & 0.92 & $(0.61-1.39)$ & 0.705 & 1.19 & $(0.77-1.83)$ & 0.427 \\
\hline Obese & $4 / 499$ & 0.80 & 0.49 & $(0.18-1.33)$ & 0.161 & 0.74 & $(0.27-2.05)$ & 0.562 \\
\hline \multicolumn{9}{|c|}{ Stroke/non-CNS SE/MI } \\
\hline Underweight & $15 / 540$ & 2.78 & 2.11 & $(1.20-3.70)$ & 0.009 & 1.64 & $(0.90-2.99)$ & 0.104 \\
\hline Normal & $63 / 4,392$ & 1.43 & 1.00 & Reference & & 1.00 & Reference & \\
\hline Overweight & $33 / 2,161$ & 1.53 & 1.06 & $(0.69-1.61)$ & 0.800 & 1.19 & $(0.76-1.84)$ & 0.447 \\
\hline Obese & $7 / 497$ & 1.41 & 0.97 & $(0.44-2.12)$ & 0.940 & 1.15 & $(0.52-2.57)$ & 0.727 \\
\hline
\end{tabular}

$B M I$ body mass index, $C H F$ congestive heart failure, $C I$ confidence interval, $C N S$ central nervous system, $C r C l$ creatinine clearance, $H R$ hazard ratio, $M I$ myocardial infarction, $S E$ systemic embolism, TIA transient ischemic attack

${ }^{a}$ BMI categories $\left(\mathrm{kg} / \mathrm{m}^{2}\right)$ : underweight, $<18.5$; normal, 18.5 to $<25$; overweight, 25 to $<30$; and obese, $\geq 30$

${ }^{\mathrm{b}}$ Adjusted for age ( $\geq 75 /<75$ years), sex (female/male), $\mathrm{CrCl}(<50 / \geq 50 \mathrm{ml} / \mathrm{min})$, initial dose (10 mg/15 mg), hypertension (yes/no), diabetes mellitus (yes/no), CHF (yes/no), prior ischemic stroke/TIA (yes/no), vascular disease (yes/no), hepatic dysfunction (yes/no), and oral antiplatelet use (yes/no) 
Table 4 Adverse events leading to all-cause mortality

\begin{tabular}{|c|c|c|c|c|}
\hline & \multicolumn{4}{|l|}{$\mathrm{BMI}\left(\mathrm{kg} / \mathrm{m}^{2}\right)$} \\
\hline & $\begin{array}{l}\text { Underweight } \\
(<18.5) \\
(n=542)\end{array}$ & $\begin{array}{l}\text { Normal } \\
(18.5 \text { to }<25) \\
(n=4410)\end{array}$ & $\begin{array}{l}\text { Overweigh } \\
(25 \text { to }<30) \\
(n=2167)\end{array}$ & $\begin{array}{l}\text { Obese } \\
(\geq 30) \\
(n=499)\end{array}$ \\
\hline Adverse events & 44 & 72 & 33 & 4 \\
\hline \multicolumn{5}{|l|}{ MedDRA System Organ Class ${ }^{\mathrm{a}}$} \\
\hline Cardiac disorders ${ }^{\mathrm{b}}$ & $12(2.21)$ & $13(0.29)$ & $8(0.37)$ & $1(0.20)$ \\
\hline Cancer $^{\mathrm{b}}$ & $9(1.66)$ & $15(0.34)$ & $5(0.23)$ & $1(0.20)$ \\
\hline Respiratory disorders $^{\mathrm{b}}$ & $8(1.48)$ & $12(0.27)$ & $5(0.23)$ & $0(0)$ \\
\hline Infections $^{\mathrm{b}}$ & $5(0.92)$ & $14(0.32)$ & $2(0.09)$ & $2(0.40)$ \\
\hline General and whole-body disorders & $3(0.55)$ & $6(0.14)$ & $5(0.23)$ & $0(0)$ \\
\hline Renal disorders & $2(0.37)$ & $1(0.02)$ & $0(0)$ & $0(0)$ \\
\hline Nervous system disorders & $1(0.18)$ & $7(0.16)$ & $5(0.23)$ & $0(0)$ \\
\hline Metabolism and nutrition disorders & $1(0.18)$ & $4(0.09)$ & $1(0.05)$ & $0(0)$ \\
\hline $\begin{array}{l}\text { Injury, poisoning, and procedural } \\
\text { complications }\end{array}$ & $1(0.18)$ & $3(0.07)$ & $1(0.05)$ & $0(0)$ \\
\hline Vascular disorders & $0(0)$ & $0(0)$ & $2(0.09)$ & $0(0)$ \\
\hline
\end{tabular}

Data are presented as $n(\%)$. MedDRA terms with $\geq 2$ events in either BMI categories are shown $B M I$ body mass index, MedDRA Medical Dictionary for Regulatory Activities

${ }^{\text {a }}$ Based on MedDRA version 20.0

${ }^{\mathrm{b}}$ The terms used in MedDRA are as follows: cancer, neoplasms benign, malignant, and unspecified (including cysts and polyps); infections, infections and infestations; respiratory disorders, respiratory, thoracic, and mediastinal disorders
non-CNS SE/MI; fourth, in multivariable analyses, being underweight was found to be an independent predictor of all-cause mortality.

Limited conclusions could be drawn from previous global studies investigating the impact of BMI on outcomes in patients with AF receiving anticoagulants because very few patients included in these analyses were underweight [7, 12-14]. The efficacy and safety of rivaroxaban in patients with NVAF were established in the global Rivaroxaban Once Daily Oral Direct Factor Xa Inhibition Compared with Vitamin K Antagonism for Prevention of Stroke and Embolism Trial in Atrial Fibrillation (ROCKET AF) and Japan-specific J-ROCKET AF phase 3 studies [15, 16]. In a post hoc analysis of the ROCKET AF study, high BMI was associated with reduced stroke risk, whereas bleeding risk was similar across BMI categories (18.50-24.99, 25.00-29.99, and $\left.\geq 30 \mathrm{~kg} / \mathrm{m}^{2}\right)$ [17]. These data suggest that BMI is associated with efficacy and safety outcomes in patients receiving rivaroxaban for stroke prevention in NVAF; however, there were limited data in patients who are underweight (i.e. BMI $<18.5 \mathrm{~kg} /$ $\mathrm{m}^{2}$ ), because these patients were excluded from the post hoc analysis and constituted only $\sim 1 \%$ of the ROCKET AF population [15]. Although J-ROCKET AF may have included a greater proportion of patients who were underweight than ROCKET AF, sample sizes were too small to evaluate the influence of BMI on clinical outcomes. A meta-analysis of studies that included Asian patients with AF concluded that being underweight was associated with an increased risk of stroke or SE, cardiovascular death, and all-cause death, whereas in all patients with AF, being overweight or obese was not associated with increased risks of these outcomes, data that support the existence of the obesity paradox [5]. However, this study did not evaluate potential associations between BMI and bleeding outcomes. Data from another recent metaanalysis that included Western and Asian patients also supported the existence of the obesity paradox; however, this study included patients with body weight $<60 \mathrm{~kg}$ in the $\mathrm{BMI}<18.5 \mathrm{~kg} / \mathrm{m}^{2}$ group if BMI information was not available, which may not properly reflect differences in body composition between Western and Asian patients [18]. Previous studies in Japanese patients with AF have suggested that those who were underweight had worse prognosis than those of normal weight, but these studies were conducted during the warfarin era $[6,19]$.

The XAPASS was conducted to evaluate the real-world safety and effectiveness of rivaroxaban in patients with NVAF [8]. The 1-year follow-up results in 9578 patients demonstrated low incidences of bleeding and thromboembolic events, suggesting that rivaroxaban is safe and effective for stroke prevention in daily clinical practice [9]. Compared with previous studies, such as the ROCKET AF post hoc analysis, the present sub-analysis of the XAPASS included a population with more diverse demographic characteristics and a greater proportion of patients were underweight 
$\left(\mathrm{BMI}<18.5 \mathrm{~kg} / \mathrm{m}^{2}\right)$. The XAPASS dataset was therefore more suitable for the evaluation of potential associations between BMI and clinical outcomes in patients receiving rivaroxaban than the ROCKET AF post hoc analysis.

Results from the present XAPASS sub-analysis in Japanese patients with NVAF receiving rivaroxaban showed that BMI was not independently associated with the incidence of major bleeding even though the incidence of major bleeding in patients who were underweight was numerically higher than in those of normal weight. Similar results were reported in the J-RHYTHM registry sub-analysis, which showed a trend towards increasing rates of major hemorrhage with decreasing BMI, but that being underweight was not associated with a significantly increased risk of hemorrhage compared with patients of normal weight [6]. However, Park et al. demonstrated that patients who were underweight had a significantly increased risk of major bleeding compared with those of normal weight [7]. These inconsistencies may be due to differences in study design (e.g. observational or retrospective), sample size, and class of oral anticoagulant administered (e.g. warfarin or DOAC).

Data from the present study demonstrated no significant association between BMI and the incidence of thromboembolism, which is consistent with results from previous studies [6, 7]. However, a significantly higher incidence of stroke/non-CNS SE/MI was observed in patients who were underweight than in those of normal weight, which may be partially explained by differences in demographic characteristics between BMI groups. For example, patients who were underweight tended to be older and have lower $\mathrm{CrCl}$ values than patients in the other BMI categories, and these factors have previously been shown to be independently associated with increased risk of thromboembolism in Japanese patients [20, 21]. Furthermore, although $\mathrm{CHADS}_{2}$ scores were similar across the BMI categories, different patterns of comorbidities in patients who were underweight (i.e. a greater proportion with prior ischemic stroke/TIA compared with the other BMI groups) may have had an impact on thromboembolic outcomes. Indeed, analysis of the Fushimi AF Registry demonstrated that previous stroke/TIA and chronic kidney disease were strong independent risk factors for cardioembolic stroke in patients with $\mathrm{AF}$ even after adjustment for $\mathrm{CHADS}_{2}$ score components [22]. It should be noted that the proportion of patients who received an underdose of rivaroxaban in the present study was lowest $(17.9 \%)$ in the underweight group and highest $(32.5 \%)$ in the obese group (Supplementary Fig. 1); therefore, underdosing is unlikely to account for the increased incidence of thromboembolic events in patients who were underweight.

The finding from the present study that being underweight was independently associated with increased allcause mortality is in agreement with results from studies conducted during the warfarin era [6] and the DOAC era
[7, 23], and may be suggestive of a lean paradox rather than an obesity paradox [24]. Patients who are underweight tend to be susceptible to poor nutritional status [25], which may lead to becoming ill. Previous studies have concluded that increased mortality in patients who are underweight could, at least in part, be caused by residual confounding from pre-existing disease leading to weight loss [26]. Indeed, one hypothesis is that the obesity paradox in patients with AF may be due to confounding variables such as lead time bias. Patients who are underweight may be less likely to be tested for cardiovascular diseases than patients with obesity, and therefore, as a result, may receive a diagnosis only when the disease is more advanced, by which time prognosis is worse [27]. In particular, the current analysis revealed higher percentages of mortality from cancer, respiratory disorders, and infections in the underweight patient group compared to the other groups (Table 4). This result is consistent with a community-based cohort study targeting Japanese patients over 80 years old, in that mortality from cardiovascular disorders, cancer, or pneumonia tended to be highest in the underweight group compared with other groups, even though the differences did not reach statistical significance [28]. Those real-world data on causes of mortality in the underweight AF patients in the XAPASS study could be important information for physicians, because these multimorbid patients are often excluded in randomized clinical trials.

There are some limitations of this sub-analysis. First, BMI information was missing for 1960 patients (20.5\%), which might have affected the results. Second, it is difficult to generalize the present findings to patients with NVAF receiving rivaroxaban worldwide, because the current study used doses approved in Japan (15 mg or $10 \mathrm{mg}$ o.d.), which differ from doses used globally ( $20 \mathrm{mg}$ or $15 \mathrm{mg}$ o.d.). Also, the median BMI of the XAPASS $\left(23.6 \mathrm{~kg} / \mathrm{m}^{2}\right)$ was lower than that of the ROCKET AF study $\left(28.3 \mathrm{~kg} / \mathrm{m}^{2}\right)$. Third, the XAPASS is a single-arm, open-label observational study. It is impossible to directly compare the outcomes of rivaroxaban treatment with those of other treatments such as warfarin and other DOACs, as previously described [9]. Fourth, the sub-analysis included only 1 year of follow-up, limiting the ability to assess for late clinical events. Fifth, selection bias by physicians in prescribing rivaroxaban and loss of patients to follow-up might have led to underestimation of the event rates. Despite these limitations, we believe that our findings may help physicians make patient-specific decisions when prescribing rivaroxaban, especially for patients who are underweight or obese.

In this sub-analysis in Japanese patients with NVAF receiving rivaroxaban, BMI was not independently associated with major bleeding and thromboembolic outcomes, whereas it was independently associated with all-cause 
mortality. Given that this sub-analysis showed a high incidence of thromboembolic events and all-cause mortality in patients who were underweight, careful management of NVAF status and comorbidities may be required in this population.

Acknowledgements The authors acknowledge the EPS Corporation for data management and analysis. Editorial support was provided by Emma Bolton DPhil of Oxford PharmaGenesis, Oxford, UK.

Funding This study was funded by Bayer Yakuhin, Ltd. (Osaka, Japan). The sponsor was involved in study design, in the collection, analysis, and interpretation of data, in writing the report, and in the decision to submit the article for publication.

\section{Compliance with ethical standards}

Conflict of interest YM, TI, SO, TK, JN, KM, and SM were advisory board members for Bayer Yakuhin, Ltd. YM received research grants from Bayer Yakuhin, Ltd., Boehringer Ingelheim, and Daiichi Sankyo, and honoraria from Bayer Yakuhin, Ltd., Boehringer Ingelheim, Bristol-Myers Squibb, and Daiichi Sankyo. TI received research grants from Bayer Yakuhin, Ltd., Bristol-Myers Squibb, Daiichi Sankyo, Medtronic Japan, and St. Jude Medical, and honoraria from Bayer Yakuhin, Ltd., Bristol-Myers Squibb, Daiichi Sankyo, Ono, and Pfizer; TI was an advisory board member for Bristol-Myers Squibb. TK received a research grant from Bayer Yakuhin, Ltd. JN received a research grant from Nihon Medi-Physics. KM received honoraria from Astellas, AstraZeneca, Bayer Yakuhin, Ltd., BMS, Boehringer Ingelheim, Daiichi Sankyo, Japan Stryker, Kowa, Mitsubishi-Tanabe, Nihon Medi-Physics, Nippon Chemiphar, Otsuka, Pfizer, Sawai, and Sumitomo Dainippon; KM was an advisory board member for CSL Behring and Medico's Hirata. SM received research grants from Astellas, Brainlab, Bristol-Myers Squibb, Carl Zeiss Meditec, Chugai, CSL Behring, Daiichi Sankyo, Eisai, Medtronic, Meiji, Mitsubishi-Tanabe, MSD, Mizuho, Nihon Medi-Physics, Otsuka, Pfizer, Philips Electronics Japan, Sanofi, Siemens Healthcare, and Takeda. YH, YK, YO, TS, SS, and SY are employees of Bayer Yakuhin, Ltd.

Open Access This article is licensed under a Creative Commons Attribution 4.0 International License, which permits use, sharing, adaptation, distribution and reproduction in any medium or format, as long as you give appropriate credit to the original author(s) and the source, provide a link to the Creative Commons licence, and indicate if changes were made. The images or other third party material in this article are included in the article's Creative Commons licence, unless indicated otherwise in a credit line to the material. If material is not included in the article's Creative Commons licence and your intended use is not permitted by statutory regulation or exceeds the permitted use, you will need to obtain permission directly from the copyright holder. To view a copy of this licence, visit http://creativecommons.org/licenses/by/4.0/.

\section{References}

1. Haas S, Camm AJ, Bassand JP, Angchaisuksiri P, Cools F, Corbalan R, Gibbs H, Jacobson B, Koretsune Y, Mantovani LG, Misselwitz F, Panchenko E, Ragy HI, Stepinska J, Turpie AG, Sawhney JP, Steffel J, Lim TW, Pieper KS, Virdone S, Verheugt FW, Kakkar AK (2019) Predictors of NOAC versus VKA use for stroke prevention in patients with newly diagnosed atrial fibrillation: results from GARFIELD-AF. Am Heart J 213:35-46

2. Badheka AO, Rathod A, Kizilbash MA, Garg N, Mohamad T, Afonso L, Jacob S (2010) Influence of obesity on outcomes in atrial fibrillation: yet another obesity paradox. Am J Med 123(7):646-651

3. Wang J, Yang YM, Zhu J, Zhang H, Shao XH, Tian L, Huang B, Yu LT, Gao X, Wang M (2014) Overweight is associated with improved survival and outcomes in patients with atrial fibrillation. Clin Res Cardiol 103(7):533-542

4. Miyasaka Y, Barnes ME, Gersh BJ, Cha SS, Bailey KR, Abhayaratna WP, Seward JB, Tsang TS (2006) Secular trends in incidence of atrial fibrillation in Olmsted County, Minnesota, 1980 to 2000, and implications on the projections for future prevalence. Circulation 114(2):119-125

5. Zhu W, Wan R, Liu F, Hu J, Huang L, Li J, Hong K (2016) Relation of body mass index with adverse outcomes among patients with atrial fibrillation: a meta-analysis and systematic review. J Am Heart Assoc 5(9):e004006

6. Inoue $\mathrm{H}$, Kodani $\mathrm{E}$, Atarashi $\mathrm{H}$, Okumura $\mathrm{K}$, Yamashita $\mathrm{T}$, Origasa $H$ (2016) Impact of body mass index on the prognosis of Japanese patients with non-valvular atrial fibrillation. Am J Cardiol 118(2):215-221

7. Park CS, Choi EK, Kim HM, Lee SR, Cha MJ, Oh S (2017) Increased risk of major bleeding in underweight patients with atrial fibrillation who were prescribed non-vitamin K antagonist oral anticoagulants. Heart Rhythm 14(4):501-507

8. Ogawa S, Minematsu K, Ikeda T, Kitazono T, Nakagawara J, Miyamoto S, Murakawa Y, Ohashi Y, Takeichi M, Okayama Y, Yamanaka S, Inuyama L (2018) Design and baseline characteristics of the Xarelto post-authorization safety \& effectiveness study in japanese patients with atrial fibrillation (XAPASS). J Arrhythm 34(2):167-175

9. Ikeda T, Ogawa S, Kitazono T, Nakagawara J, Minematsu K, Miyamoto S, Murakawa Y, Takeichi M, Ohashi Y, Okayama Y, Sunaya T, Yamanaka S (2019) Real-world outcomes of the Xarelto postauthorization safety \& effectiveness study in Japanese patients with atrial fibrillation (XAPASS). J Cardiol 74(1):60-66

10. WHO Consultation on Obesity (1999: Geneva, Switzerland) \& World Heath Organization (2000). Obesity: preventing and managing the flobal epidemic: report of a WHO consultation. https:// apps.who.int/iris/handle/10665/42330. Accessed 2 Aug 2019

11. Schulman S, Kearon C (2005) Definition of major bleeding in clinical investigations of antihemostatic medicinal products in non-surgical patients. J Thromb Haemost 3(4):692-694

12. Boriani G, Ruff CT, Kuder JF, Shi M, Lanz HJ, Rutman H, Mercuri MF, Antman EM, Braunwald E, Giugliano RP (2019) Relationship between body mass index and outcomes in patients with atrial fibrillation treated with edoxaban or warfarin in the ENGAGE AF-TIMI 48 trial. Eur Heart J 40(19):1541-1550

13. Netley J, Howard K, Wilson W (2019) Effects of body mass index on the safety and effectiveness of direct oral anticoagulants: a retrospective review. J Thromb Thrombol 48(3):359-365

14. Tittl L, Endig S, Marten S, Reitter A, Beyer-Westendorf I, BeyerWestendorf J (2018) Impact of BMI on clinical outcomes of NOAC therapy in daily care-results of the prospective Dresden NOAC Registry (NCT01588119). Int J Cardiol 262:85-91

15. Patel MR, Mahaffey KW, Garg J, Pan G, Singer DE, Hacke W, Breithardt G, Halperin JL, Hankey GJ, Piccini JP, Becker RC, Nessel CC, Paolini JF, Berkowitz SD, Fox KA, Califf RM (2011) Rivaroxaban versus warfarin in nonvalvular atrial fibrillation. $\mathrm{N}$ Engl J Med 365(10):883-891

16. Hori M, Matsumoto M, Tanahashi N, Momomura S, Uchiyama S, Goto S, Izumi T, Koretsune Y, Kajikawa M, Kato M, Ueda H, Iwamoto K, Tajiri M (2012) Rivaroxaban vs warfarin in Japanese 
patients with atrial fibrillation-the J-ROCKET AF study. Circ J 76(9):2104-2111

17. Balla SR, Cyr DD, Lokhnygina Y, Becker RC, Berkowitz SD, Breithardt G, Fox KAA, Hacke W, Halperin JL, Hankey GJ, Mahaffey KW, Nessel CC, Piccini JP, Singer DE, Patel MR (2017) Relation of risk of stroke in patients with atrial fibrillation to body mass index (from Patients Treated With Rivaroxaban and Warfarin in the Rivaroxaban Once Daily Oral Direct Factor Xa Inhibition Compared with Vitamin K Antagonism for Prevention of Stroke and Embolism Trial in Atrial Fibrillation Trial). Am J Cardiol 119(12):1989-1996

18. Zhou Y, Ma J, Zhu W (2019) Efficacy and safety of direct oral anticoagulants versus warfarin in patients with atrial fibrillation across BMI categories: a systematic review and meta-analysis. Am J Cardiovasc Drugs. https://doi.org/10.1007/s40256-01900362-4

19. Yanagisawa S, Inden Y, Yoshida N, Kato H, Miyoshi-Fujii A, Mizutani Y, Ito T, Kamikubo Y, Kanzaki Y, Hirai M, Murohara $\mathrm{T}$ (2016) Body mass index is associated with prognosis in Japanese elderly patients with atrial fibrillation: an observational study from the outpatient clinic. Heart Vessels 31(9):1553-1561

20. Kodani E, Atarashi H, Inoue H, Okumura K, Yamashita T, Origasa H (2018) Impact of creatinine clearance on outcomes in patients with non-valvular atrial fibrillation: a subanalysis of the J-RHYTHM Registry. Eur Heart J Qual Care Clin Outcomes 4(1):59-68

21. Yamashita Y, Hamatani Y, Esato M, Chun YH, Tsuji H, Wada H, Hasegawa K, Abe M, Lip GYH, Akao M (2016) Clinical characteristics and outcomes in extreme elderly (age $\% 3 \mathrm{e} /=85$ years) Japanese patients with atrial fibrillation: the Fushimi AF Registry. Chest 149(2):401-412

22. Yasuda K, Fukuda S, Nakamura M, Ohtani R, Kuwata Y, Takata M, Sainouchi M, Gotou M, Masuda Y, Kawarazaki S, Kawabata Y, Murase N, Aoki T, Yonemoto N, Akao M, Tsukahara T (2018) Predictors of cardioembolic stroke in Japanese patients with atrial fibrillation in the Fushimi AF Registry. Cerebrovasc Dis Extra $8(2): 50-59$
23. Wang L, Du X, Dong JZ, Liu WN, Zhou YC, Li SN, Guo XY, Jiang CX, Yu RH, Sang CH, Tang RB, Long DY, Liu N, Bai R, Macle L, Ma CS (2019) Body mass index and all-cause mortality in patients with atrial fibrillation: insights from the China atrial fibrillation registry study. Clin Res Cardiol. https://doi. org/10.1007/s00392-019-01473-3

24. Elagizi A, Kachur S, Lavie CJ, Carbone S, Pandey A, Ortega FB, Milani RV (2018) An overview and update on obesity and the obesity paradox in cardiovascular diseases. Prog Cardiovasc Dis 61(2):142-150

25. Buys DR, Roth DL, Ritchie CS, Sawyer P, Allman RM, Funkhouser EM, Hovater M, Locher JL (2014) Nutritional risk and body mass index predict hospitalization, nursing home admissions, and mortality in community-dwelling older adults: results from the UAB Study of Aging with 8.5 years of follow-up. J Gerontol A Biol Sci Med Sci 69(9):1146-1153

26. Aune D, Sen A, Prasad M, Norat T, Janszky I, Tonstad S, Romundstad P, Vatten LJ (2016) BMI and all cause mortality: systematic review and non-linear dose-response meta-analysis of 230 cohort studies with 3.74 million deaths among 30.3 million participants. BMJ 353:i2156

27. De Schutter A, Lavie CJ, Milani RV (2014) The impact of obesity on risk factors and prevalence and prognosis of coronary heart disease-the obesity paradox. Prog Cardiovasc Dis 56(4):401-408

28. Takata Y, Ansai T, Soh I, Akifusa S, Sonoki K, Fujisawa K, Awano S, Kagiyama S, Hamasaki T, Nakamichi I, Yoshida A, Takehara T (2007) Association between body mass index and mortality in an 80-year-old population. J Am Geriatr Soc 55(6):913-917

Publisher's Note Springer Nature remains neutral with regard to jurisdictional claims in published maps and institutional affiliations. 